\title{
DESIGN OF ECONOMICALLY OPTIMAL ZERO-DEFECT ACCEPTANCE SAMPLING WITH RECTIFICATION WHEN DIAGNOSIS ERRORS ARE PRESENT
}

\author{
Roberto da Costa Quinino * \\ Departamento de Estatística / ICEX \\ Universidade Federal de Minas Gerais (UFMG) \\ Belo Horizonte - MG \\ roberto@est.ufmg.br
}

\author{
Linda Lee Ho \\ Departamento de Eng. de Produção / EP \\ Universidade de São Paulo (USP) \\ São Paulo - SP \\ lindalee@usp.br

\section{Emílio Suyama} \\ Departamento de Estatística / ICEX \\ Universidade Federal de Minas Gerais (UFMG) \\ Belo Horizonte - MG \\ suyama@est.ufmg.br \\ * Corresponding author/autor para quem as correspondências devem ser encaminhadas \\ Recebido em 12/2003; aceito em 02/2005 após 1 revisão \\ Received December 2003; accepted February 2005 after one revision
}

\begin{abstract}
In this paper we present the optimum sampling size in zero-defect acceptance with rectification sampling scheme in the presence of misclassification errors. Its development is based on an economical model. The procedures are implemented in a program using the software Matlab and illustrated by an example.
\end{abstract}

Keywords: zero-defect acceptance sampling; diagnosis errors; cost function; rectification.

\section{Resumo}

Neste trabalho determinamos o tamanho ótimo amostral em uma amostragem de aceitação zerodefeitos com retificação na presença de erros de diagnóstico. O desenvolvimento é baseado em um modelo econômico. Os procedimentos são implementados no Matlab e ilustrados através de um exemplo numérico.

Palavras-chave: amostragem de aceitação zero defeitos; erros de classificação; função custo; retificação. 


\section{Introduction}

Consider manufactured items evaluated by a sampling scheme known as zero-defect acceptance with rectification. That is, in $T$ lots, each one having $N$ products, a sample of size $m$ is extracted from each. If all sampled items after non-destructive inspection are classified as conforming, then the lot is accepted. Otherwise all items (sampled and non-sampled) are inspected, the non-conforming ones are rectified (i.e. all non-conforming units may be replaced or discarded after $100 \%$ inspection) and then the lot is accepted. Such a procedure is known as zero-defect sampling with rectification. This type of sampling scheme is frequently used when manufacturing costs are very high, for example in the manufacturing of semiconductors. Figure 1 illustrates such a procedure.

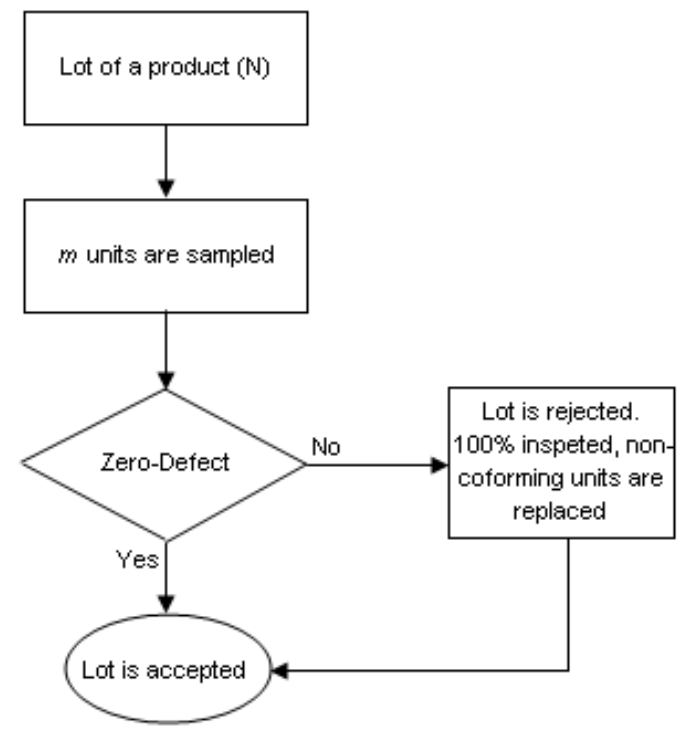

Figure 1 - Acceptance sampling: zero defect with rectification.

Papers about zero-defect sampling with rectification can be found in the literature. We may mention the contributions from Hahn (1986), Brush et al. (1990), Greenberg \& Stokes (1992) and Anderson et al. (2001). In them, the main objective is to present an estimator for the number of non-conforming items in such a sampling scheme. Anderson et al. (2001) introduced the possibility of misclassification errors in this type of acceptance sampling. That means one item is evaluated as non-conforming but in reality it is conforming, or an item is classified as conforming but it is in fact non-conforming. About diagnosis errors, many authors have made contributions in this direction. For an early example, Johnson et al. (1991) pointed out that the diagnosis errors can endanger the performance of an acceptance sampling. Minton (1972) provided expressions to analyze the effect of inefficient inspection and has proposed corrections on the power of single sampling inspection plans, mainly in misclassifications of defectives as non-defectives. Different authors have presented methodologies to minimize the impact of diagnosis errors in the acceptance sampling. We cite Greenberg \& Stokes (1995), Markowski \& Markowski (2002), Quinino \& Ho (2004) and Quinino \& Suyama (2002). 
The use of an economical model to plan acceptance sampling is not a new idea, but it is still a subject of great interest as Wetherill \& Chiu (1975) pointed out. It was recently used in Ferrel \& Chhoker (2002) to determine the producer's tolerance that minimizes the producer's loss and consumer's loss in a single sampling, with inspection and non-inspection procedures using a quadratic function to describe the consumer's cost. Aminzadeh (2003) actually used the Inverse Gaussian distribution as a lifetime model to obtain optimal values for sample size and action limit employing economic variable acceptance-sampling plans based on a steploss function. Starbird (1997) has specified the condition under which zero-defect with rectification is the policy that minimizes the supplier's expected annual cost.

In this paper, we consider the determination of an economically optimum sample size $m$ that minimizes the cost function in zero-defect acceptance sampling with rectification in the presence of misclassification errors. The inclusion of diagnosis errors in the determination of sample size in this sampling procedure is a natural extension of the earlier papers mentioned. Economical models mentioned in the literature do not include the possibility of the diagnosis errors and rectification. The components of cost function include inspection cost, costs due to the presence of non-conforming items in accepted lots and costs due to diagnosis errors.

In Section 2, we introduce the notation and hypothesis considered in this paper. The expected cost function and the procedure to determine the optimum value of $m$ is developed in Section 3. As this probabilistic model of sampling process can be viewed as a Markov chain, the description of the absorbing and/or transient states and their transition probability matrices is presented in Section 4. The procedure is illustrated by a numerical example in Section 5 and we finish this paper with discussions and extensions for future research.

\section{Notation and Hypothesis}

Consider a lot with $N$ units and a random sample of $m$ units selected without replacement. $D$ is the number of non-conforming units in the lot and it is a binomial random variable $(N, p)$, with probability $\pi$, or it is equal to zero with probability $(1-\pi)$. So the probability of all items being conforming $(D=0)$ is given by $\pi(1-p)^{N}+(1-\pi)$. This family of distributions is flexible enough to show a good fit to observed distributions related to the quality of a lot with appropriate choice of the probability $\pi$, allowing a simple interpretation and leading to a simple theory (Hald, 1981).

Let:

$e_{1} \rightarrow$ the probability of a conforming item being classified as non-conforming;

$e_{2} \rightarrow$ the probability of a non-conforming item being classified as conforming;

$c_{0} \rightarrow$ the cost to inspect an item;

$c_{1} \rightarrow$ the cost of a non-conforming item in the accepted lot;

$c_{2} \rightarrow$ the cost of judging erroneously an item as non-conforming when it is conforming;

$D_{1} \rightarrow$ the number of actual non-conforming items in the initial sample of size $m$ in the lot;

$D_{2} \rightarrow$ the number of actual non-conforming items in $(N-m)$ non-sampled items in the lot;

$D=D_{1}+D_{2} \rightarrow$ the number of non-conforming items in the lot; 
$Y_{1} \rightarrow$ the number of items declared non-conforming after inspection in the initial sample of size $m$ in the lot;

$Y_{2} \rightarrow$ the number of items declared non-conforming in $(N-m)$ non-sampled items in the lot if the lot were rectified;

$Y=Y_{1}+Y_{2} \rightarrow$ the number of items that would be declared as non-conforming in lot if the lot were rectified;

$D_{l} \mid D \rightarrow$ denotes the conditioned distribution of $D_{l}$ on $D$ and it is assumed to be Hypergeometric $(m, D, N)$.

\section{Cost Function}

This Section develops the expected cost function per lot $\left(E_{m}\right)$ employing the earlier notations and hypothesis from Section 2. The expected cost function is composed of three parts. The first one $\left(E_{m}^{1}\right)$ is related to costs of inspection of $m$ items and the possibility of inspecting the $(N-m)$ non-sampled items. The latter is conditioned to the event $\left(Y_{1}>0\right)$ (at least one non-conforming item in the $m$ initial inspected items) so, it follows that:

$$
E_{m}^{1}=c_{0} m+c_{0}(N-m) P\left(Y_{1}>0\right) .
$$

The second component $\left(E_{m}^{2}\right.$ ) is due to the possibility of an item being classified as conforming when it is non-conforming. Such a result can produce differences in expenses when the lot is accepted or when it is rejected in the inspection. Figure 2 illustrates such a procedure.

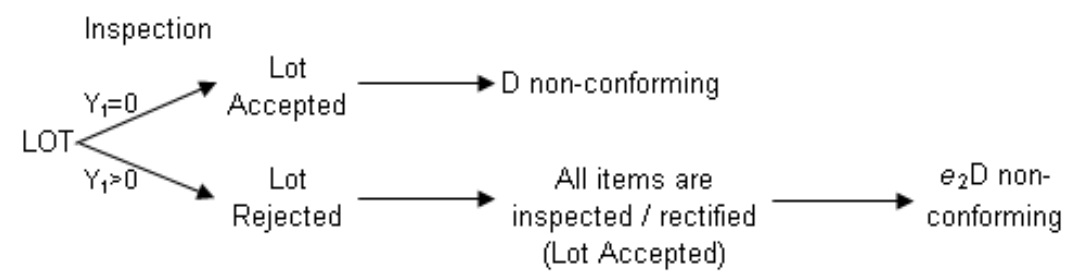

Figure 2 - The number of non-conforming when the lot is accepted/rejected.

Thus, resulting in

$$
E_{m}^{2}=c_{1} E\left[I_{\left[Y_{1}=0\right]} D+e_{2} I_{\left[Y_{1}>0\right]} D\right]
$$

where $I_{[\bullet]}$ denotes an indicator function; $E(\bullet)$, the expected value of a random variable.

The last part $\left(E_{m}^{3}\right.$ ) is due to the consequence of classifying an item as non-conforming when it is a conforming item. In this case, the lot is rejected, consequently all items inspected and there is a chance of it being rectified unnecessarily:

$$
E_{m}^{3}=c_{2} e_{1} E\left[(N-D) I_{\left[Y_{1}>0\right]}\right] .
$$


So the expected cost $\left(E_{m}\right)$ is $E_{m}=E_{m}^{1}+E_{m}^{2}+E_{m}^{3}$. Specifically,

$$
\begin{aligned}
& E_{m}=c_{0} m+c_{0}(N-m) P\left(Y_{1}>0\right)+c_{1} E\left[I_{\left[Y_{1}=0\right]} D+e_{2} I_{\left[Y_{1}>0\right]} D\right]+c_{2} e_{1} E\left[(N-D) I_{\left[Y_{1}>0\right]}\right] \\
& =c_{0} m+c_{0}(N-m) P\left(Y_{1}>0\right)+c_{1} E\left[\left(1-I_{\left[Y_{1}>0\right]}\right) D+e_{2} I_{\left[Y_{1}>0\right]} D\right]+c_{2} e_{1} E\left[(N-D) I_{\left[Y_{1}>0\right]}\right] \\
& =c_{0} m+\left[c_{0}(N-m)+c_{2} e_{1} N\right] P\left(Y_{1}>0\right)+c_{1} E[D]-\left[c_{1}\left(1-e_{2}\right)+c_{2} e_{1}\right] E\left[I_{\left[Y_{1}>0\right]}\left(D_{1}+D_{2}\right)\right]
\end{aligned}
$$

with

- $P\left(Y_{1}>0\right)=1-P\left(Y_{1}=0\right)=1-\left\{\pi E\left[E\left[P\left(Y_{1}=0 \mid D_{1}\right) \mid D\right]\right]+\left(1-e_{1}\right)^{m}(1-\pi)\right\}$;

and $E\left[E\left[P\left(Y_{1}=0 \mid D_{1}\right) \mid D\right]\right]=\sum_{D=0}^{N} \sum_{D_{1}=0}^{\min (m, D)} \frac{\left(\begin{array}{l}D \\ D_{1}\end{array}\right)\left(\begin{array}{l}N-D \\ m-D_{1}\end{array}\right)}{\left(\begin{array}{l}N \\ m\end{array}\right)}\left(\begin{array}{l}N \\ D\end{array}\right) p^{D}(1-p)^{N-D}\left(1-e_{1}\right)^{m-D_{1}} e_{2}^{D_{1}}$

- $E[D]=\pi N p$

- $E\left[I_{\left[Y_{1}>0\right]} D_{1}\right]=\pi E\left[E\left[D_{1} P\left(Y_{1}>0 \mid D_{1}\right) \mid D\right]\right]=\pi\left\{1-E\left[E\left[D_{1} P\left(Y_{1}=0 \mid D_{1}\right) \mid D\right]\right]\right\} ;$

- $E\left[I_{\left[Y_{1}>0\right]} D_{2}\right]=\pi E\left[D_{2}\right] E\left[E\left[P\left(Y_{1}>0 \mid D_{1}\right) \mid D\right]\right]$ as $D_{2}$ and $P\left(Y_{1}>0\right)$ are conditionally independent on $D_{1}$.

with $E\left[D_{2}\right]=\pi(N-m) p$;

and

$E\left[E\left[D_{1} P\left(Y_{1}>0 \mid D_{1}\right) \mid D\right]\right]=\sum_{D=0}^{N} \sum_{D_{1}=0}^{\min (m, D)} \frac{\left(\begin{array}{l}D \\ D_{1}\end{array}\right)\left(\begin{array}{l}N-D \\ m-D_{1}\end{array}\right)}{\left(\begin{array}{l}N \\ m\end{array}\right)}\left(\begin{array}{l}N \\ D\end{array}\right) p^{D}(1-p)^{N-D}\left[1-\left(1-e_{1}\right)^{m-D_{1}} e_{2}^{D_{1}}\right] D_{1}$

with $E(\bullet \mid \bullet) \rightarrow$ denoting the conditioned expectation value.

\section{Markov Chains in Zero-Defect Acceptance Sampling with Rectification in the Presence of Diagnosis Errors}

The processes of sampling and inspection and the decision to accept or reject the lot after the inspection presented in Section 3 can be modeled as a non-irreducible Markov chain with transition matrix $\boldsymbol{P}$. The idea consists of sampling a single item systematically each time until a total of $m$ units are extracted. The set of states can be denoted by the vector $(s, j, k, t, z)$, such that $s+j+k+t=z ; z=0, \ldots, m$, are absorbing or transient states. The variable $s$ can be viewed as the number of conforming items correctly classified as conforming; $j$ is the number of conforming items incorrectly judged as non-conforming; $k$ is the number of nonconforming items classified as conforming; $t$ is the number of non-conforming items 
correctly judged as non-conforming and $z$ the number of items evaluated. As the aim was to calculate the probability of a lot being accepted or rejected, the states $(j=t=0$ and $z=m)$ or $(j=1$ or $t=1)$ were considered as absorbing. They indicate respectively that the inspection procedure of the $m$ items is finished or the lot was accepted or rejected even before the $m$ inspections are finished. In this context it is necessary to examine only states for which $j \leq 1$ and $t \leq 1$.

Consider the probabilities in the transition matrix $\boldsymbol{P}$ conditioned on the random variable $D$. $\boldsymbol{P}_{\boldsymbol{1}}$ is the transition matrix conditioned on $D$ when it follows a binomial distribution with parameters $(N, p)$ and $\boldsymbol{P}_{\mathbf{2}}$ the transition matrix when $\mathrm{D}=0$. The matrix $\boldsymbol{P}_{\boldsymbol{1}}$ occurs with probability $\pi$ and the matrix $\boldsymbol{P}_{2}$ with probability $(1-\pi)$.

The probabilities in the transition matrices $\boldsymbol{P}_{\boldsymbol{1}}$ and $\boldsymbol{P}_{\mathbf{2}}$, related to the inspected lot are respectively:

$$
\begin{gathered}
\boldsymbol{P}_{1} \rightarrow\left\{\begin{array}{l}
P[(s+1, j, k, t, z+1) \mid(s, j, k, t, z)]=A_{1} \\
P[(s, j+1, k, t, z+1) \mid(s, j, k, t, z)]=A_{2} \\
P[(s, j, k+1, t, z+1) \mid(s, j, k, t, z)]=A_{3} \\
P[(s, j, k, t+1, z+1) \mid(s, j, k, t, z)]=A_{4} \\
P[(s, j, k, t, z) \mid(s, j, k, t, z)]=1, \text { if } z=m \text { or } j=1 \text { or } t=1 \\
\text { Otherwise }=0
\end{array}\right. \\
\boldsymbol{P}_{2} \rightarrow\left\{\begin{array}{l}
P[(s+1, j, 0,0, z+1) \mid(s, j, 0,0, z)]=\left(1-e_{1}\right) \\
P[(s, j+1,0,0, z+1) \mid(s, j, 0,0, z)]=e_{1} \\
P[(s, j, k, t, z) \mid(s, j, k, t, z)]=1, \text { if } z=m \text { or } j=1 \text { or } t=1 \quad k>0 \\
\text { Otherwise }=0
\end{array}\right.
\end{gathered}
$$

where

$$
\begin{gathered}
A_{1}=\left(1-e_{1}\right) \sum_{D=k}^{N-s} \frac{N-z-D+k}{N-z} \times \frac{B}{C} ; \\
A_{2}=e_{1} \sum_{D=k}^{N-s} \frac{N-z-D+k}{N-z} \times \frac{B}{C} ; \\
A_{3}=e_{2} \sum_{D=k}^{N-s} \frac{D-k}{N-z} \times \frac{B}{C}
\end{gathered}
$$

and

$$
A_{4}=\left(1-e_{2}\right) \sum_{D=k}^{N-s} \frac{D-k}{N-z} \times \frac{B}{C} .
$$


where

$$
B=\left(\begin{array}{l}
N \\
D
\end{array}\right) p^{D}(1-p)^{N-D} \frac{\left(\begin{array}{l}
D \\
k
\end{array}\right)\left(\begin{array}{c}
N-D \\
z-k
\end{array}\right)}{\left(\begin{array}{c}
N \\
z
\end{array}\right)}
$$

is the probability of a lot containing $\mathrm{D}$ non-conforming items and $z$ items being inspected with $k$ non-conforming and $s$ conforming ones and

$$
C=\sum_{D=k}^{N-s}\left(\begin{array}{l}
N \\
D
\end{array}\right) p^{D}(1-p)^{N-D} \frac{\left(\begin{array}{l}
D \\
k
\end{array}\right)\left(\begin{array}{c}
N-D \\
z-k
\end{array}\right)}{\left(\begin{array}{l}
N \\
z
\end{array}\right)}
$$

is the probability of $z$ items being inspected with $k$ non-conforming and $s$ conforming ones.

The probability of each state after $m$ inspections is given by the row vectors $\boldsymbol{P}_{\boldsymbol{I}}{ }^{(m)}=\boldsymbol{P}^{0} \boldsymbol{P}_{\boldsymbol{I}}{ }^{m}$ and $\boldsymbol{P}_{\mathbf{2}}{ }^{(m)}=\boldsymbol{P}^{0} \boldsymbol{P}_{\mathbf{2}}{ }^{m}$, where $\boldsymbol{P}^{0}=[1,0,0 \ldots \ldots, 0]$ is the vector of the probabilities of initial state and the probability is equal to one for the state $(s=0, j=0, k=0, t=0, z=0)$ and equal to zero for other states. Each element of $\boldsymbol{P}^{0}, \boldsymbol{P}_{\boldsymbol{1}}{ }^{(m)}$ and $\boldsymbol{P}_{\mathbf{2}}{ }^{(m)}$ is associated with one state $(s, j, k, t, z)$. The non-null probabilities in row vectors $\boldsymbol{P}_{\boldsymbol{1}}{ }^{(m)}$ and $\boldsymbol{P}_{\mathbf{2}}{ }^{(m)}$ indicate absorbing states and the decision of acceptance or rejection of the lot.

Making $\boldsymbol{P}^{(m)}=\pi \boldsymbol{P}_{\mathbf{1}}^{(m)}+(1-\pi) \boldsymbol{P}_{\mathbf{2}}^{(m)}$, it is possible to calculate easily the probabilities of interest, mainly the one related to the acceptance of the lot. For example, summing up the probabilities of the absorbing states $(s, j, k, t, z)$ such that $j=0, t=0$ and $z=m$ will provide the probability of accepting a lot [This is denoted by $\left.P\left(Y_{l}=0\right)\right]$. The probability of non-acceptance of a lot is given by the sum of the probabilities of the absorbing states $(s, j, k, t, z)$, when $j=1$ or $t=1$ in $\boldsymbol{P}^{(m)}$. States with $k>0$ will indicate the wrong acceptance of the lot and states with $j=1$ indicate that the lot was rejected wrongly.

It is interesting to observe that the probabilities of a lot being correctly accepted, wrongly accepted, correctly rejected and wrongly rejected can be provided employing Markov chain properties but this approach is computationally intensive in this context since the number of states depends on the sample size $m$.

\section{Determination of the Optimum Sample Size $m^{\circ}$}

The optimum value of $m\left(m^{\circ}\right)$ is the one that minimizes (3.1) and it can be obtained by direct search substituting values of $m=0, \ldots, N$ in (3.1). As $N$ is usually a large number, a direct search can be a difficult task, demanding too much time. A limit $L_{1} \leq N$ is proposed to speed up the search. Whether accepting or rejecting the lot, the cost to inspect $m$ items will be at least $c_{0} m$. For the optimum value $m^{\circ}$, this will be $c_{0} m^{\circ}$. However, if the 
inspection is not performed, that is, when $m=0$, the expected cost will be $E_{m=0}=N p \pi c_{1}$. As $m^{\circ} c_{0}<E_{m^{\circ}} \leq E_{m=0}$ then it follows that $m^{\circ} \leq N p \pi c_{1} / c_{0}$. So a direct search to find $m^{\circ}$ must be proceeded for all integer values of $m$ satisfying $m \leq L_{1}=\min \left\{N ; N p \pi c_{1} / c_{0}\right\}$.

If $m \leq 0.1 N$, the hypergeometric distribution can be approximated by a binomial distribution (Johnson, 1994) and this approach simplifies the mathematical modeling. So a new expression for $E_{m}$, denoted by $E_{m}^{\Delta}$, can be derived when this approximation is used for the random variable $\mathrm{D}_{1}$. In this sense, a new boundary built for $E_{m}^{\Delta}$ can be employed to search for the optimum sample size. To find a boundary for $E_{m}^{\Delta}$ may not be an easy task; a conditioned boundary on $p_{h}=D / N$ for $E_{m}^{\Delta}$ will be proposed to simplify the task and then, a new limit can be established for $E_{m}$ using this result.

Let $E_{m}^{*}$ be the cost function of $E_{m}^{\Delta}$ when $p_{h}=D / N$ and $m^{\bullet}$ is its optimum sample size. Examining $\Delta E_{m^{*}}^{*}=E_{m^{*}}^{*}-E_{m^{*}-1}^{*} \leq 0$ and after some algebraic manipulation, the inequality

$$
a-\pi b^{m^{\bullet}-1} l-\left(N-m^{\bullet}\right) b^{m^{\bullet}-1} k \leq 0
$$

can be stated with

$$
\begin{gathered}
a=\left(c_{0}+N c_{2} e_{1}^{2}\right)(1-\pi) ; \\
b=p_{h} e_{1} /\left(1-e_{1}\right)+\left(1-p_{h}\right) ; \\
l=\left[p_{h} c_{1}\left(1-e_{2}\right)+p_{h} c_{2} e_{1}\right]\left[\left(n e_{2}-1\right)\left(e_{1}+p_{h}\left(1-e_{1}-e_{2}\right)+\left(1-e_{2}\right)\right] /\left[1-e_{1}-p_{h}\left(1-e_{1}-e_{2}\right)\right]-\right. \\
{\left[\mathrm{c}_{0}+N c_{2} e_{1}\left(e_{1}+p_{h}\left(1-e_{1}-e_{2}\right)\right)\right] ;}
\end{gathered}
$$

and

$$
\begin{aligned}
k= & {\left[\pi p_{h} c_{1}\left(1-e_{2}\right)+\pi p_{h} c_{2} e_{1}\right]\left[\left(1-p_{h}\right)\left(1-e_{1}-e_{2}\right)\left(e_{1}+p_{h}\left(1-e_{1}-e_{2}\right)\right)\right] /\left[1-e_{1}-p_{h}\left(1-e_{1}-e_{2}\right)\right]-} \\
& {\left[\pi \mathrm{c}_{0}\left(e_{1}+p_{h}\left(1-e_{1}-e_{2}\right)\right]\right.}
\end{aligned}
$$

A set of inequalities expressed in (5.2) can be obtained from (5.1) as functions of $k$ and $l$.

$$
\left\{\begin{array}{l}
a-\pi b^{m^{\bullet}-1} l-\left(N-m^{\bullet}\right) b^{m^{\bullet}-1} k \geq 0, \text { if } l \leq 0 \text { and } k \leq 0 \\
a-N b^{m^{\bullet}-1} k \leq 0, \text { if } l<0 \text { and } k>0 \\
a-\pi b^{m^{\bullet}-1} l \leq 0, \text { if } l>0 \text { and } k<0 \\
a-\pi b^{m^{\bullet}-1} l-N b^{m^{\bullet}-1} k \leq 0, \text { if } l>0 \text { and } k>0
\end{array}\right.
$$

And from (5.2), a boundary $L_{2}$ for $m^{\bullet}$ is proposed in (5.3) 


$$
L_{2} \rightarrow\left\{\begin{array}{l}
m^{\bullet}=0, \text { if } l \leq 0 \text { and } k \leq 0 \\
m^{\bullet} \leq 1+\frac{\log \frac{a}{N k}}{\log b}, \text { if } l \leq 0 \text { and } k>0 \\
m^{\bullet} \leq 1+\frac{\log \frac{a}{\pi l}}{\log b}, \text { if } l>0 \text { and } k \leq 0 \\
m^{\bullet} \leq 1+\frac{\log \frac{a}{\pi l+N k}}{\log b}, \text { if } l>0 \text { and } k>0
\end{array}\right.
$$

Negative values on the right side of the inequality (5.3) mean $m^{\bullet}=0$. The expression (5.3) indicates that values lower than the boundary will yield $\Delta E_{m}^{*} . \leq 0$. This indicates the existence of one and only one minimum value of $E_{m}^{*}$ for values lower than the boundary and that it is also the global minimum. The limit expressed in (5.3) is conditioned on $p_{h}$ and examining all possible values of $p_{h}$ a new boundary for $E_{m}^{\Delta}$ expressed as $L_{3}=\max \left\{L_{2}\left(p_{h}\right)\right\}, p_{h}=D / N, D=1, \ldots, N$ can be proposed. This would be for integers lower than $L_{3}, \Delta E_{m^{\Delta}}^{\Delta}=E_{m^{\Delta}}^{*}-E_{m^{\Delta}-1}^{*} \leq 0$, where $m^{\Delta}$ denotes the optimum value. This means that there is one and only one extreme value for $E_{m}^{\Delta}$ lower than this boundary and it is also the global minimum. Moreover, the condition $m \leq 0.1 \mathrm{~N}$ guarantees a good approximation of a hypergeometric distribution by a binomial distribution. So, this additional condition must also be verified, that is, if $L_{3} \leq 0.1 \mathrm{~N}$. In this case, the boundary $L_{3}$ can be employed to delimit a minimum for $E_{m}$ which will be $m^{\Delta}$.

The strategy can be drawn to perform a computational search for the optimum value $m\left(\mathrm{~m}^{\circ}\right)$ for the expression (3.1). If $L_{1} \leq 0.1 \mathrm{~N}$ then search all integers lower than $\min \left\{L_{1} ; L_{3}\right\}$, until reaching the minimum value $m^{\circ}$. If $L_{1}>0.1 N$ then search all integers lower than $\min \left\{0.1 N ; L_{3}\right\}$ until reaching the minimum value. Compare this result with the result of search among integers higher than $0.1 \mathrm{~N}$ but lower or equal to $L_{1}$. The value of $m^{\circ}$ is the lowest. Note that the computational search is performed searching only integer values, starting always with the lower one. The flowchart in Figure 3 illustrates the decision process described in this section. 


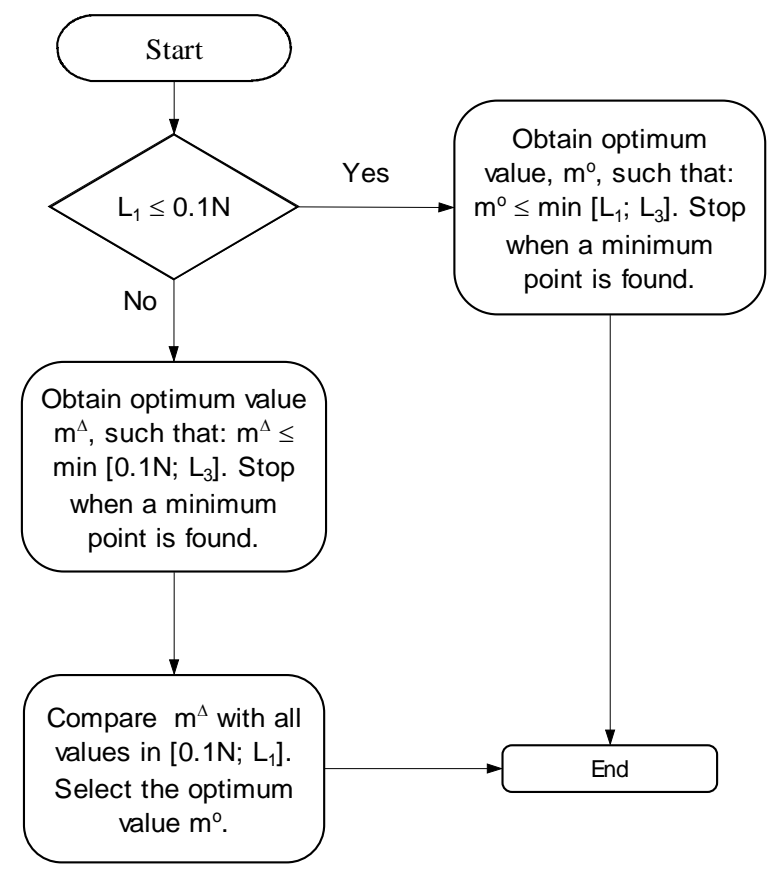

Figure 3 - The decision process of the optimum value.

\section{Numerical Example and Discussions}

The example described in this section is based on Hahn (1986), Greenberg (1992), Greenberg (1995) and Anderson (2001). Consider lots with $N=5000$ items, each to be inspected by a zero-defect with rectification procedure. In this context consider: $\pi=0.1$; $p=0.05$ and $e_{1}=e_{2}=0.001$ and the following costs: $c_{0}=\$ 3.00, c_{1}=\$ 100.00$, $c_{2}=\$ 500.00$. The question is to find the optimum value of $m\left(\mathrm{~m}^{\circ}\right)$ that allows for minimizing (3.1). This value can be found using direct search substituting values as shown in the flowchart (Figure 2). According to the earlier results, it indicates a search for integers $0 \leq m \leq 49$ and $0.1 N=500<m \leq L_{1}=833$. In the first interval, $0 \leq m \leq 49$, the optimum value was found to be $15\left(\mathrm{~m}^{\Delta}\right)$.

A program using the software Matlab was developed (see Appendix 1) to find the optimum value $m^{\circ}$. This program provided as the optimum sample size $m^{\circ}=15$ which corresponds to an expected cost of $\$ 2360.26$. In the absence of diagnosis errors the optimum sample increases to $m^{\circ}=57$, which corresponds to an expected cost of $\$ 1707.02$. If the sample size of $m^{\circ}=57$ were wrongly employed (i.e. discarding misclassifications errors), a serious error in cost estimation would result, with the expected cost becoming $\$ 2796.50$ being $61 \%$ over the required $\$ 1707.02$. Note that even small misclassification error probabilities as $e_{1}=e_{2}=0.001$ can significantly alter the expected cost as well as the optimum sample size. To corroborate the analytical results, another program was developed using Matlab to 
simulate a zero-defect with rectification by Monte Carlo simulations with $m=15$. Five hundred thousand runs were used in this simulation and the difference between the expected cost and the analytical results was lower than $0.3 \%$. Figure 4 illustrates the behavior of $E_{m}$ as a function of the value of $m$.

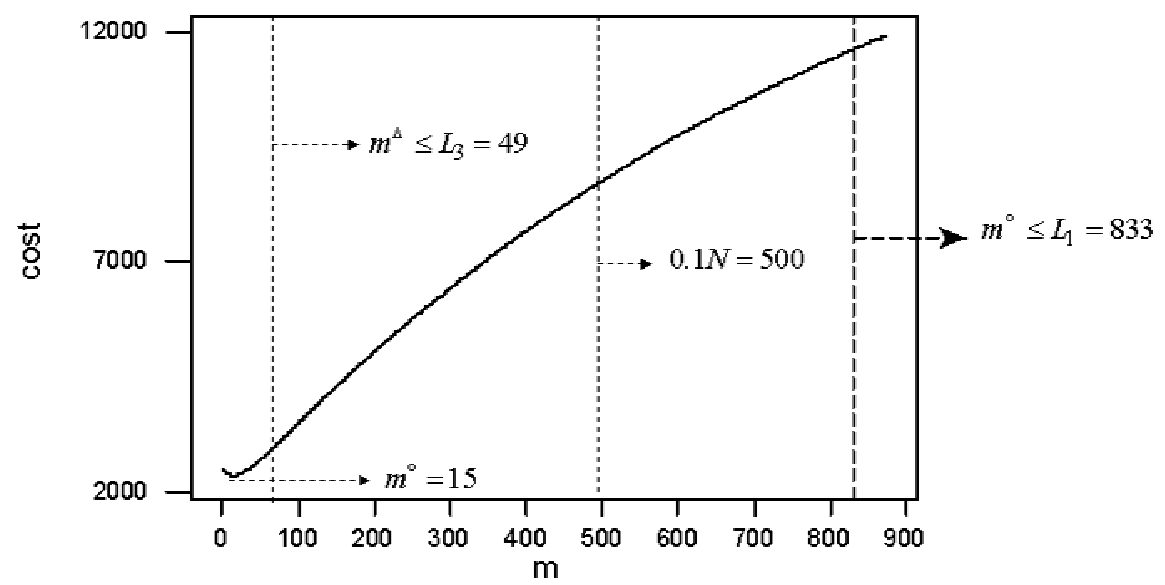

Figure 4 - Values of $m$ versus expected cost.

A sensitivity analysis was performed to evaluate the behavior of the optimum values of $m$ as functions of the parameters. Since all possible scenarios can result in a high number of possibilities to examine, and analyzing all of them can become unmanageable, here an analysis varying one parameter at a time was conducted. The ranges of the parameters explored in this case are

- $0 \leq c_{0} \leq 5$;

- $0 \leq c_{1} \leq 700$;

- $0 \leq c_{2} \leq 2900$;

- $0 \leq e_{1} \leq 0.002$;

- $0 \leq e_{2} \leq 0.15$ and

- $0 \leq p \leq 1$.

The results of this analysis are plotted in Figure 5. It was observed that as $c_{0}$ increases $m^{\circ}$ tends to zero, indicating that the best option is a non-sampling procedure. If $c_{2}$ increases, then the value of $m^{\circ}$ tends to zero, which is justified by the fact that $m^{\circ}=0$ results in the possibility of a cost $c_{2}$ being eliminated.

If $c_{1}$ increases, the value of $m^{\circ}$ tends to $N$ since total inspection in a lot decreases considerably the number of non-conforming items. This is because the probability of a nonconforming item being classified correctly is $\left(1-e_{2}\right)$ and certainly $e_{2}<0.5$ in practical situations. 
If $e_{1}$ and $e_{2}$ increase, it is not feasible to do sampling with rectification since the amount of items wrongly classified will eliminate the benefit of the proposed procedure, which is to provide an accepted lot with lower quantity of non-conforming items.

If $p \downarrow 0, m^{\circ} \downarrow 0$ since there are only conforming items in the lot. As $p \uparrow 1, m^{\circ} \downarrow 1$. This can be justified since the probability to reject the lot alters slightly when $m>1$ indicating that there is no necessity to sample more than one item.
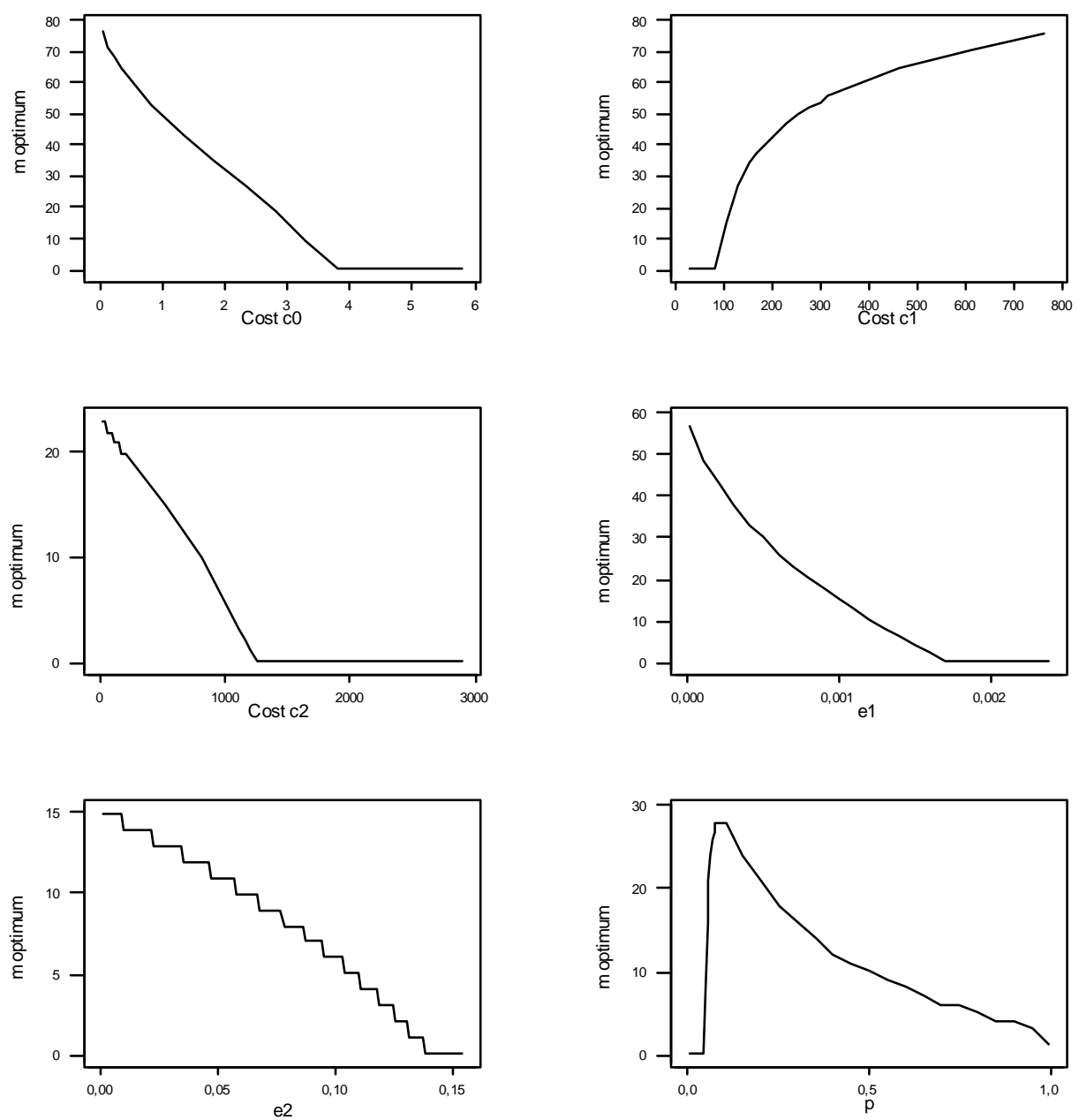

Figure 5 - Optimum values of $\mathrm{m}$ versus costs $c_{0}, c_{1}, c_{2}, p, e_{1}$, and $e_{2}$.

Now using the Markov chain approach and considering a sample size of 15 items $\left(\mathrm{m}^{\circ}\right)$, the probability of accepting the lot is 0.93227 and to rejecting the lot is 0.06773 . Possible routes to accept and to reject the lot are listed respectively in Tables 1 and 2. The vectors described in these tables are elements of $\boldsymbol{P}^{(m)}$. Such a vector allows the user to verify which absorbing states indicate the correct acceptance of the lot or the correct rejection of the lot. With the 
vector $\boldsymbol{P}^{(m)}$ it is possible to calculate conditional probabilities of interest. If the lot was accepted, the probability to be correctly accepted (only conforming items in the sample) is 0.99996. Similarly, if the lot was rejected, the probability to be correctly rejected (at least one non-conforming units in the sample) is 0.78711. These results suggest a tighter verification in the rejected lots as an attempt to decrease the number of lots wrongly rejected.

Table 1 - Absorbing states - lot accepted.

\begin{tabular}{|c|c|c|c|c|c|}
\hline $\mathbf{s}$ & $\mathbf{j}$ & $\mathbf{k}$ & $\mathbf{T}$ & $\mathbf{Z}$ & Probability \\
\hline 15 & 0 & 0 & 0 & 15 & $9,3223 \mathrm{E}-01$ \\
\hline 14 & 0 & 1 & 0 & 15 & $3,6067 \mathrm{E}-05$ \\
\hline 13 & 0 & 2 & 0 & 15 & $1,3301 \mathrm{E}-08$ \\
\hline 12 & 0 & 3 & 0 & 15 & $3,0366 \mathrm{E}-12$ \\
\hline 11 & 0 & 4 & 0 & 15 & $4,7995 \mathrm{E}-16$ \\
\hline 10 & 0 & 5 & 0 & 15 & $5,5628 \mathrm{E}-20$ \\
\hline 9 & 0 & 6 & 0 & 15 & $4,8846 \mathrm{E}-24$ \\
\hline 8 & 0 & 7 & 0 & 15 & $3,3100 \mathrm{E}-28$ \\
\hline \multicolumn{6}{|c|}{ of the values } \\
\hline
\end{tabular}

Table 2 - Absorbing states - lot rejected.

\begin{tabular}{|c|c|c|c|c|c|}
\hline $\mathbf{S}$ & $\mathbf{J}$ & $\mathbf{K}$ & $\mathbf{t}$ & $\mathbf{z}$ & Probability \\
\hline 0 & 0 & 0 & 1 & 1 & 4,9950E-03 \\
\hline 1 & 0 & 0 & 1 & 2 & $4,7405 \mathrm{E}-03$ \\
\hline 2 & 0 & 0 & 1 & 3 & 4,4990E-03 \\
\hline 3 & 0 & 0 & 1 & 4 & $4,2698 \mathrm{E}-03$ \\
\hline 4 & 0 & 0 & 1 & 5 & $4,0522 \mathrm{E}-03$ \\
\hline . & . & . & . & . & . \\
\hline . & . & . & . & . &. \\
\hline . & . & . & . & . & . \\
\hline 6 & 0 & 7 & 1 & 14 & $5,0000 \mathrm{E}-30$ \\
\hline 5 & 0 & 7 & 1 & 13 & $2,0000 \mathrm{E}-30$ \\
\hline 0 & 1 & 6 & 0 & 7 & $1,0000 \mathrm{E}-30$ \\
\hline 4 & 0 & 7 & 1 & 12 & $1,0000 \mathrm{E}-30$ \\
\hline \multicolumn{5}{|c|}{ Sum of the probabilities } & $6,7731 \mathrm{E}-02$ \\
\hline
\end{tabular}

Another point of interest is to evaluate the probability of rejecting a lot of only conforming items $(r p)$ or the probability accepting a lot of at least one non-conforming unit $(a d)$. The probability of observing only conforming units in a lot is given by $\pi(1-p)^{N}+(1-\pi)$ but usually large lots are preferable (Montgomery, 2001) and $\pi(1-p)^{N} \cong 0$ is assumed to be 
true, so the sum of the probabilities indicating acceptance of the lot according to vector $\boldsymbol{P}_{\mathbf{1}}{ }^{(m)}$ is 0.00036 and rejection of the lot according $\boldsymbol{P}_{\mathbf{2}}{ }^{(m)}$ is 0.015 . These values correspond respectively to the conditional probabilities of accepting a lot given at least one nonconforming item in a lot and to of rejecting the lot given only conforming items in a lot.

Consider the probability of finding only conforming units in a lot approximately equal to 0.9 . In this case, $r p=0.013$ and $a d=0.000036$ but values of $e_{1}$ and $e_{2}$ can significantly alter these probabilities. For example, $e_{1}=e_{2}=0.005$, they correspond $r p=0.065$ and $a d=0.00171$. However, it is important to keep in mind that the approach presented here is economical and that high values of $r p$ and $a d$ are strong indications of inadequate attributions for the costs. Another plausible solution is to try a semi-economical approach sometimes called statistical-economical with the goal of minimizing functions like (3.1) restricted to maximum values of $r p$ and $a d$, for example.

\section{Conclusions and Final Remarks}

Diagnosis errors can cause a significant impact in determining the optimum sample size in a zero-defect with rectification procedure. Even small diagnosis error probabilities, such as $e_{1}=0.001$ and $e_{2}=0.001$ can significantly alter the value of optimum $m\left(m^{\circ}\right)$, as illustrated in this study. Therefore, diagnosis errors must be incorporated in the model and evaluated from an economic perspective.

Extensions of this study can be made in two directions. One is to change the initial criteria in the sampling inspection for a value other than zero, that is $c, c \geq 0$. Another alternative is to make repetitive tests to minimize the effect of the diagnosis errors. An item would be classified as conforming if the number of conforming independent classifications is higher than a specified value $a$. In this situation, the objective is to determine the optimum values of $m$, the number of the independent repetitive inspections for an item, the value of $a$ and the value of $c$ in order to minimize the total expected cost.

\section{Acknowledgment}

The authors would like to acknowledge the many valuable comments of an anonymous reviewer.

\section{References}

(1) Aminzadeh, M.S. (2003). Bayesian Economic Variable Acceptance-Sampling Plan Using Inverse Gaussian Model and Step-Loss Function. Communication in Statistics Theory and Methods, 32(5), 961-982.

(2) Anderson, M.A.; Greenberg, B.S. \& Stokes, S.L. (2001). Acceptance sampling with rectification when inspection errors are present. Journal of Quality Technology, 33(4), 493-505. 
(3) Brush, G.G.; Hoadley, B. \& Saperstein, B. (1990). Estimating outgoing quality using the quality measurement plan. Technometrics, 32, 31-41.

(4) Ferrell, G.W. \& Chhoker, A. (2002). Design of economically optimal acceptance sampling plans with inspection error. Computers \& Operations Research, 29, 1283-1300.

(5) Greenberg, B.S. \& Stokes, S.L. (1992). Estimating nonconformance rates after zero defect sampling with rectification. Technometrics, 34(2), 203-213.

(6) Greenberg, B.S. \& Stokes, S.L. (1995). Repetitive testing in the presence of inspection errors. Technometrics, 37(1), 102-111.

(7) Hahn, G.J. (1986). Estimating the percent nonconforming in the accepted product after zero defect sampling. Journal of Quality Technology, 18(3), 182-188.

(8) Hald, A. (1967). The determination of single sampling attribute plans with given producer's and consumer's risk. Technometrics, 9, 401-415.

(9) Hald, A. (1981). Statistical Theory of Sampling Inspection by Attributes. Academic Press, New York.

(10) Johnson, N.L.; Kotz, S. \& Balakrishnan, N. (1992). Univariate Discrete Distributions. Second Edition, John Wiley \& Sons, Inc., New York.

(11) Johnson, N.L.; Kotz, S. \& Wu, X. (1991). Inspection errors for attributes in quality control. Chapman \& Hall, London.

(12) Markowski, E.P. \& Markowski, C.A. (2002). Improved attribute acceptance sampling plans in the presence of misclassification error. European Journal of Operation Research, 139(3), 501-510.

(13) Minton, G. (1972). Verification error in single sampling inspection plans for processing survey data. Journal of the American Statistical Association, 67, 46-54.

(14) Montgomery, D.C. (2001). Introduction to statistical quality control. 4th ed., John Wiley \& Sons, Inc.

(15) Quinino, R.C. \& Ho, L.L. (2004). Repetitive tests as an economic alternative procedure to control attributes with diagnosis errors. European Journal of Operation Research, 155, 209-225.

(16) Quinino, R.C. \& Suyama, E. (2002). Número ótimo de classificações independentes com erro na avaliação da conformidade de produtos. Pesquisa Operacional, 22(1), $1-8$.

(17) Starbird, A. (1997). Acceptance sampling, imperfect production, and the optimality of zero defects. Naval Research Logistics, 44, 515-530.

(18) Wetherill, G.B. \& Chiu, W.K. (1975). A review of acceptance sampling schemes with emphasis on the economic aspect. International Statistical Review, 43(2), 91-210. 


\section{Appendix}

Use a Matlab Editor to write the files *.m. Run optimum.m the software Matlab.

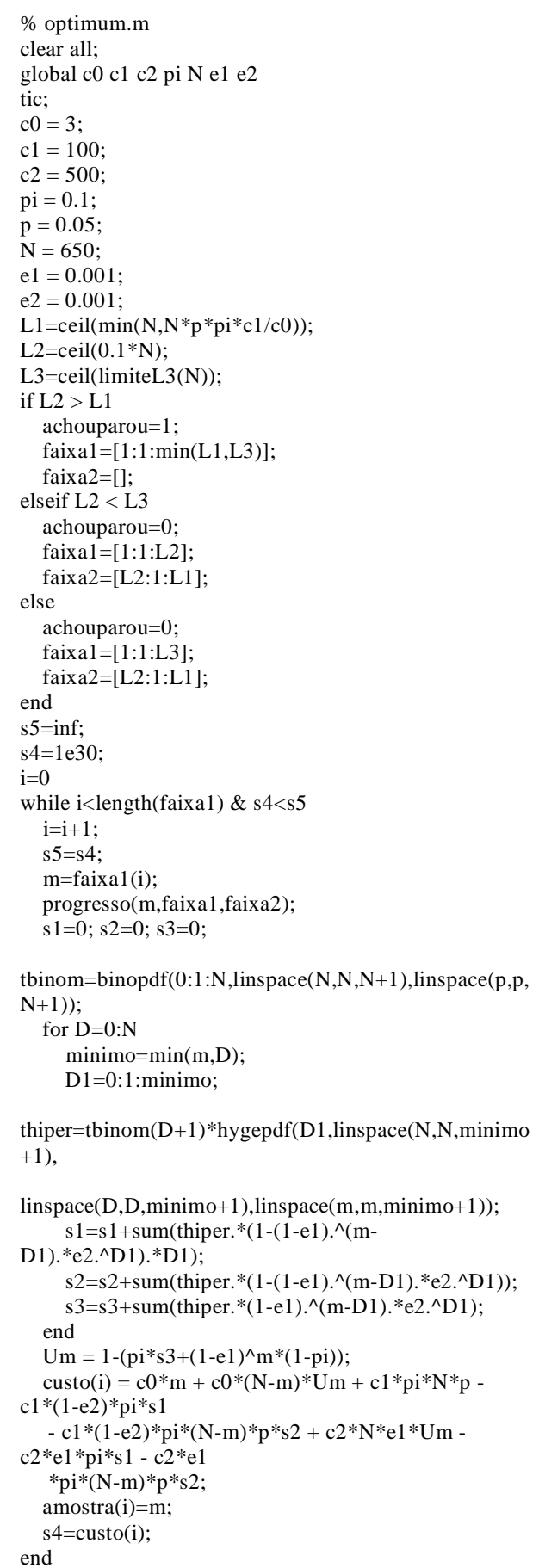

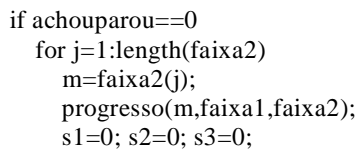

tbinom=binopdf( $(0: 1: \mathrm{N}$, linspace $(\mathrm{N}, \mathrm{N}, \mathrm{N}+1)$, linspace $(\mathrm{p}, \mathrm{p}$, $\mathrm{N}+1)$ );

for $\mathrm{D}=0: \mathrm{N}$

$\operatorname{minimo}=\min (\mathrm{m}, \mathrm{D})$

D1=0:1:minimo;

thiper=tbinom $(\mathrm{D}+1) *$ *hygepdf $(\mathrm{D} 1$, linspace $(\mathrm{N}, \mathrm{N}$, minimo $+1)$

linspace (D,D,minimo+1), linspace $(\mathrm{m}, \mathrm{m}, \operatorname{minimo}+1))$; $\mathrm{s} 1=\mathrm{s} 1+\mathrm{sum}\left(\right.$ thiper.* $*\left(1-(1-\mathrm{e} 1) .^{\wedge}(\mathrm{m}-\right.$

D1).*e2.^D1).*D1);

$\mathrm{s} 2=\mathrm{s} 2+\operatorname{sum}\left(\right.$ thiper. $*(1-(1-\mathrm{e} 1))^{\wedge}(\mathrm{m}-$

D1).*e2.^D1));

$\mathrm{s} 3=\mathrm{s} 3+\mathrm{sum}\left(\right.$ thiper. $\left.*(1-\mathrm{e} 1) .^{\wedge}(\mathrm{m}-\mathrm{D} 1) . *^{*} \mathrm{e} 2 .^{\wedge} \mathrm{D} 1\right)$;

end

$\mathrm{Um}=1-\left(\mathrm{pi} * \mathrm{~s} 3+(1-\mathrm{e} 1)^{\wedge} \mathrm{m} *(1-\mathrm{pi})\right)$;

custo $(\mathrm{i}+\mathrm{j})=\mathrm{c} 0 * \mathrm{~m}+\mathrm{c} 0 *(\mathrm{~N}-\mathrm{m}) * \mathrm{Um}+\mathrm{c} 1 * \mathrm{pi} * \mathrm{~N} * \mathrm{p}-$ c1*(1-e2)*pi*s1 -

$\mathrm{c} 1 *(1-\mathrm{e} 2) * \mathrm{pi} *(\mathrm{~N}-\mathrm{m}) * \mathrm{p} * \mathrm{~s} 2+\mathrm{c} 2 * \mathrm{~N} * \mathrm{e} 1 * \mathrm{Um}-$

$\mathrm{c} 2 * \mathrm{e} 1 * \mathrm{pi} * \mathrm{~s} 1$ -

$\mathrm{c} 2 * \mathrm{e} 1 * \mathrm{pi}(\mathrm{N}-\mathrm{m}) * \mathrm{p} * \mathrm{~s} 2$

$\operatorname{amostra}(i+j)=m$;

end

end

amostra $=[0$ amostra $]$

custo $=[\mathrm{N} * \mathrm{p} * \mathrm{pi} * \mathrm{c} 1$ custo $]$

$[$ Minimo, pos $]=\min$ ( custo)

Optimum $=\operatorname{amostra}($ pos $)$;

clc;

fprintf('\%60s $\backslash \mathrm{n}$ ', '*********************************

**************************************************

$* * * * * * * * * ')$;

fprintf('\%60s $\backslash n$ ',

Result');

fprintf('\%50s $\backslash \mathrm{n}$ ', '*******************************

***********************************************

$* * * * * * * * * * ')$

fprintf('\%2s $\backslash$ n',' ')

fprintf('\%40sไt \%10.6fln','Expected Cost

',Minimo);

fprintf('\%40s $\ \% 6.0 f \backslash n ', '$ m optimum

',Optimum);

fprintf('\%2s\n',' ');

fprintf('\%40sıt \%4.6fln',' Time (min)

',toc/60);

fprintf('\%2s $\backslash n$ ',' ');

fprintf('\%50s $\backslash \mathrm{n}$ ', '********************************

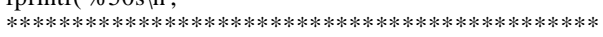

$* * * * * * * * * ')$; 